\title{
HER2-Positive Neuroendocrine Breast Cancer: Case Report and Review of Literature
}

\author{
Arpine Gevorgyan $^{a} \quad$ Giacomo Bregni $^{\mathrm{b}} \quad$ Giulia Gallia $^{\mathrm{a}}$ Elisa Zanardi ${ }^{\mathrm{b}} \quad$ Filippo de Braud $^{\mathrm{a}}$ \\ Serena Di Cosimo ${ }^{a}$
}

a Department of Medical Oncology, Fondazione IRCCS, Istituto Nazionale Tumori, Milan, Italy;

${ }^{b}$ Medical Oncology Unit 1, IRCCS AOU San Martino-IST, Genoa, Italy

\section{Established Facts}

- Neuroendocrine breast carcinoma is an established yet rare pathological finding with no specific cytotoxic treatment schedule.

\section{Novel Insights}

- Trastuzumab therapy can be effective in countering neuroendocrine breast carcinoma growth.

\section{Keywords}

Neuroendocrine carcinoma - Breast carcinoma

Trastuzumab

\section{Summary}

Background: Neuroendocrine carcinoma is an uncommon histology for breast cancer. Case Report: Our patient underwent right quadrantectomy for a neuroendocrine carcinoma in 1984 and had a bone relapse 30 years later. After thorough pathological and immunohistochemical analysis the diagnosis was confirmed and HER2 amplification was observed. Here we discuss the management, rationale and results of HER2-targeted therapy in advanced neuroendocrine breast carcinoma.

(C) 2016 S. Karger GmbH, Freiburg

\section{Introduction}

Breast cancer is one of the most frequent neoplasms in women worldwide. The most common histologies are ductal and lobular carcinoma, while neuroendocrine features are rarely diagnosed. Breast carcinomas are routinely tested for endocrine receptors, Ki67 rate and HER2 positivity to plan systemic treatment. Most recurrences are known to occur in the first decade after primary treatment. Here we report a patient with breast cancer shown histopathologically to be a neuroendocrine carcinoma (NEC), who had a bone relapse after 30 years.

\section{Case Report}

We describe a 65 -year-old woman with a past history of breast cancer. Diagnosed with breast carcinoma in 1984 when she was 36 years old, she underwent right quadrantectomy and node dissection. Final histopathological examination revealed NEC with no node involvement (N0), with a low mitotic index (Ki 67: $5 \%$ ) and pT1cNo according to TNM classification. No carcinoid associated syndrome was present. Radiotherapy without any systemic treatment was per-

\section{KARGER}

(c) 2016 S. Karger GmbH, Freiburg 
Fig. 1. a Hematoxylin and eosin-stained (H\&E) section of primary breast cancer, 1984 (1) signedout as 'malignant carcinoid'. Note the presence of an in situ component with same cytological details (2) and positivity of Grimelius silver stain (3). Morphology of relapse occurring in 1987 is strictly similar, with more pronounced pseudorosettes formation. b H\&E section of bone metastasis (1). Cancer nests showed immunohistochemical positivity for estrogen receptors $(\mathbf{2})$, very low proliferative rate with Ki $67(<5 \%, \mathbf{3})$ and $2+$ score of HER2/neu (ASCO/ CAP guidelines $2013, \mathbf{4}$ ). c Biopsy of pelvic mass shows invasive carcinoma with neuroendocrine morphology (1), with intense positivity to synaptophysin (2) and chromogranin-A (2). HER2/neu was confirmed as $2+$ score (4). Note that granular positivity of chromogranin-A is very similar in distribution to the Grimelius positivity observed in 1984 .
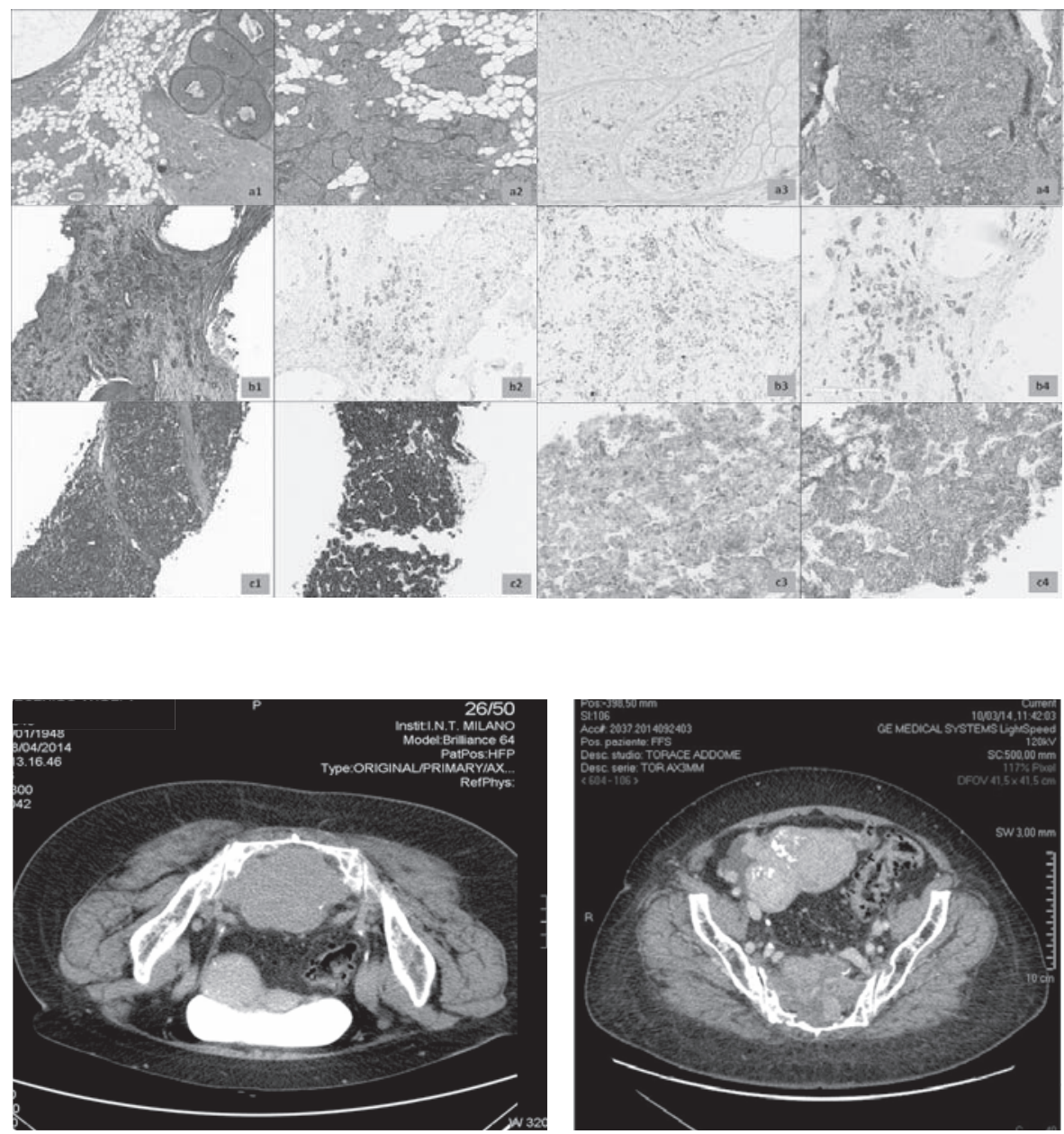

Fig. 2. Partial response after 12 weeks of treatment. Left: April 2014, right: October 2014.

formed. After a 3-year follow-up the patient underwent right mastectomy for recurrent ductal carcinoma in situ (DCIS) with invasive component (less than $<0.5 \mathrm{~mm}$ ), rpT1micNx according to TNM classification. The patient remained disease free up to March 2014 when, due to persisting back pain, the patient was seen by an oncologist. Bone scan with Tc-99m showed pathological vertebral lesions at lumbar level (D10-L1) and a pre-sacral bone mass with bone infiltration. Tumor marker CA 15-3 was $114 \mathrm{U} / \mathrm{l}$ at the time of diagnosis of metastatic bone lesions. When the patient was referred to our Institution she had already undergone biopsy of the bone lesion revealing an infiltrative carcinoma with estrogen receptor (ER)-positive, progesterone receptor (PR)- and HER2-negative breast carcinoma with Ki 67 5\%. The case was discussed with the orthopedic surgeon and a CT scan-guided sacral lesion biopsy was performed. Histopathological examination revealed breast NEC. The diagnosis was reinforced by immunohistochemistry, which showed high positivity for synaptophysin, neuron-specific enolase (NSE) and neural cell adhesion molecule (CD56). ER expression was between $66 \%$ and $100 \%$, PR expression was negative, HER2 expression was $3+$ and silver in situ hybridization (SISH) showed HER2 gene amplification. The case was discussed with the head pathologist and a review of paraffin-embedded materials from the recent bone biopsy together with initial and recurrent disease biopsy was performed (fig. 1). Our patient was started on first-line treatment with weekly paclitaxel and trastuzumab every 3 weeks for a total of 24 weeks of therapy obtaining radiological, biochemical and clinical response [1] (fig. 2). The patient had a RECIST partial disease response and since March 2015 until latest visit in October 2015 has been on maintenance treatment with trastuzumab q21 plus daily letrozole [2]. If the disease were to progress, we would favor treatment with T-DM1 [3].

\section{Review of the Literature}

Literature overview identified common patient characteristics and clinical presentations, immunohistochemical biomarkers and patients outcome. The data describe rare cases of breast NEC. Over the last 2 decades immunohistochemical studies have extended the knowledge of neuroendocrine tumors. Recent technologies have made it possible to distinguish pathological changes in endocrine cells, and it is now feasible to identify neuroendocrine morphological and biochemical features within different histological types of invasive breast cancer. However, the mere presence of neuroendocrine differentiation should not be misinterpreted as breast NEC $[2,3]$.

With the increasing knowledge of NEC characteristics, the prevalence of NEC diagnoses has dramatically dropped. The estimates reported before the introduction of new biomolecular markers were $12-19 \%$, whereas nowadays according to the WHO diagnostic criteria the reported incidence ranges from $0.3 \%$ to $0.5 \%$ [4]. 80 patients have been reported so far in peer-reviewed literature [5]. A significant proportion (59.8\%) of these cases was published after the WHO definition was established in 2003, allowing for consistency with respect to recent diagnostic criteria [6]. The mean 
age at diagnosis was 64 years (range 26-99 years; median 63 years). The mean NEC size was $32 \mathrm{~mm}$ and patients most often presented with stage II disease, indicating either large tumor size or regional lymph node metastasis at the time of diagnosis. Disease often presents a high histological grade with ER and PR positivity [1]. No large scale data are available on HER2 status in this histotype [7].

According to international guidelines no specific cytotoxic treatment schedule is available. Chemotherapy regimens for common histological types of breast cancer and pulmonary small cell carcinoma are often used for NEC. Treatment regimens reported in the literature include fluorouracil/epirubicin/cyclophosphamide combined with taxanes and platinum-based therapy; cisplatin/irinotecan; adriamycin and cytoxan or cisplatin; paclitaxel alone; and cyclophosphamide/methotrexate/fluorouracil [4].

In cases of localized disease no specific recommendations regarding surgical management are available. Treatment of these patients should be similar to that for invasive ductal carcinoma for which the choice of surgical procedure depends on the tumor's location and clinical stage. The role of biotherapy with anti-HER2 agents is underestimated due to the lack of data and lack of expression of this receptor in NEC.

\section{Conclusion}

Metastatic NEC is extremely rare and, therefore, little is known of this disease. NEC is usually thought to have quite good prognosis, but it has shown capability of aggressive behavior. The best way to identify the aggressiveness of this tumor is immunohistochemical characterization. Recently a published population-based study concluded that NEC is an aggressive variant of invasive mammary carcinoma generally diagnosed in women over 60 years of age; it usually presents with large tumor size and high histological grade, and is frequently metastatic at diagnosis. Our case shows that breast NEC can show up again after a very long disease-free period. Our patient was treated with anti-HER2 therapy which proved effective. To our knowledge this is the first case of a breast NEC being treated with trastuzumab [5]. The effectiveness of target therapy in this setting suggests that molecular assessment of the tumor may be superior to histological characterization.

\section{Disclosure Statement}

The authors declare no conflict of interest.

\section{References}

1 Wang J, Wei B, Albarracin CT, et al.: Invasive neuroendocrine carcinoma of the breast: A population-based study from the surveillance, epidemiology and end results (SEER) database. BMC Cancer 2014;14:147.

2 Sapino A, Righi L, Cassoni P, et al.: Expression of the neuroendocrine phenotype in carcinomas of the breast. Semin Diagn Pathol 2000;17:127-137.

3 Bussolati G, Gugliotta P, Sapino A, et al.: Chromogranin-reactive endocrine cells in argyrophilic carcinomas ('carcinoids') and normal tissue of the breast. Am J Pathol 1985;120:186-192.

\footnotetext{
4 Angarita FA, Rodriguez JL, Meek E, et al.: Locallyadvanced primary neuroendocrine carcinoma of the breast: Case report and review of the literature. World J Surg Oncol 2013;11:128.

5 Azzopardi JG, Muretto P, Goddeeris P, et al.: 'Carcinoid' tumours of the breast: The morphological spectrum of argyrophil carcinomas. Histopathology 1982; 6: 549-569.
}

6 Ellis P, Schnitt SJ, Sastre-Garau X: Invasive breast carcinoma, in Tavassoli FA, Devilee P (eds): Pathology and genetics: Tumors of the breast and female genita organs, 4th ed. World Health Organization, Lyon, 2003, pp. 32-34.

7 Alkaied H, Harris K, Azab B, Dai Q: Primary neuroendocrine breast cancer, how much do we know so far? Med Oncol 2012;29:2613-2618 


\section{HER2-Positive Neuroendocrine Breast Cancer: Case Report and Review of Literature}

The correct list of authors and affiliations should be as follows:

Gevorgyan A. ${ }^{\mathrm{a}}$. Bregni G. ${ }^{\mathrm{b}} \cdot$ Paolini B. ${ }^{\mathrm{c}}$ Galli G. ${ }^{\mathrm{a}} \cdot$ Zanardi E. $^{\mathrm{b}} \cdot$ de Braud F. ${ }^{\mathrm{a}}$.

Di Cosimo S. ${ }^{\mathrm{a}}$

a Department of Medical Oncology, Fondazione IRCCS, Istituto Nazionale Tumori, Milan, Italy;

${ }^{\mathrm{b}}$ Medical Oncology Unit 1, IRCCS AOU San Martino-IST, Genoa, Italy;

${ }^{\mathrm{c}}$ Department of Pathology, Fondazione IRCCS, Istituto Nazionale Tumori, Milan, Italy

The correct legend for fig. 1 should be as follows:

Fig. 1. a Hematoxylin and eosin-stained (H\&E) section of primary breast cancer, 1984 $(1,2)$ signed out as 'malignant carcinoid'. Note the presence of an in situ component with same cytological details and positivity of Grimelius silver stain (3). Morphology of relapse occurring in 1987 (4) is strictly similar, with more pronounced pseudorosettes formation. b $H \& E$ section of bone metastasis (1). Cancer nests showed immunohistochemical positivity for estrogen receptors (2), very low proliferative rate with $\mathrm{Ki} 67(<5 \%, 3)$ and $2+$ score of HER2/neu (ASCO/ CAP guidelines 2013, 4). c Biopsy of pelvic mass shows invasive carcinoma with neuroendocrine morphology (1), with intense positivity to synaptophysin (2) and chromogranin-A (2). HER2/neu was confirmed as $2+$ score (4). Note that granular positivity of chromogranin-A (3) is very similar in distribution to the Grimelius positivity observed in 1984. 